\title{
RFID-Based E-Plate with Slot-Dipole Antenna Design and Roadside Inter- rogator Deployment
}

\author{
Wenjing Zhao ${ }^{1,2, *}$, Xunguang $\mathrm{Ju}^{1,2}$ and Shuangjiang $\mathrm{Wu}^{3}$ \\ ${ }^{I}$ Department of Information and Electrical Engineering, Xuzhou Institute of Technology, Xuzhou 221000, P. R. China \\ ${ }^{2}$ Xuzhou Engineering Technology Centre of Construction Machinery Virtual Simulation, Xuzhou 221000, P. R. China \\ ${ }^{3}$ Department of Flight Support Command, Air Force Logistics College, Xuzhou 221000, P. R. China
}

\begin{abstract}
For some scenarios of vehicle tracking and identification, it is desired that radio-frequency identification (RFID) interrogators can be deployed on roadside lampposts or high poles. In order to meet this requirement, active RFID electronic license plate (e-plate) with a slot-dipole antenna is proposed to provide up-tilted beams. Numerical simulations demonstrate that the proposed e-plate antenna has desired impedance bandwidth, high gain and multi-beam radiation pattern as required at $2.45 \mathrm{GHz}$ RFID band. In addition, the estimated read range of the proposed e-plate meets the requirement of RFID system, and deployment of interrogator on roadside lamppost or high pole is analyzed.
\end{abstract}

Keywords: e-plate, interrogator deployment, radiation pattern, read range, RFID, slot-dipole antenna.

\section{INTRODUCTION}

In recent years, RFID-based e-plate has received much attention for the use in vehicle identification. Typical e-plate is an electronically-tagged self-powered number plate [1]. All vehicles equipped with e-plate can be securely identified no matter whether they are stationary or on the move. Generally speaking, an antenna, an application specific integrated circuit (ASIC) and battery are included in an active RFID tag embedded in the e-plate. The antenna plays a key role for the tag to transmit and receive RF signal. Therefore, antenna design is quite important especially when the operating frequency for RFID systems rises into the microwave region.

Antenna of e-plate design has close relationship with the material of the license plate. For the license plate of nonmetallic materials, several antenna structures have been placed behind it, such as microstrip patch antenna [2], microstrip dipole antenna [2, 3], and loop antenna [3]. However, most license plates are made of metallic materials, so that the antennas behind e-plate will not work properly. To solve this problem, slot-type antenna is a good choice, and has been successfully designed for metallic object $[4,5]$. In [6], an active e-plate has been reported by making use of meander slot to avoid damaging the layout of characters and numbers on the license plate, which limits the position and shape of the slot to a certain extent.

Thus a new active e-plate with slot-dipole antenna is introduced in this paper. The slot-dipole antenna takes a very simple structure, and it makes RFID tag module be easily integrated into the proposed e-plate. Details of the e-plate

*Address correspondence to this author at the Department of Information and Electrical Engineering, Xuzhou Institute of Technology, Xuzhou 221000, P. R. China; Tel: 15150007461; E-mail: kad_417@163.com design, simulation results, and interrogator deployment will be reported and discussed as follows.

\section{E-PLATE WITH SLOT-DIPOLE ANTENNA DE- SIGN}

The proposed license plate with a slot-dipole antenna is shown in Fig. (1). In the demonstration, the license plate is based on the structure of single-sided copper clad plate. It is composed of a dielectric substrate plate and a thin copper layer, and has dimensions of $\mathrm{L} \times \mathrm{W}=440 \times 140 \mathrm{~mm}$ (the size of Chinese standard license plate). The substrate plate may be set at the front side so that some visual information such as characters or numbers can be printed. The thin copper layer is set at the back side. On the copper side, there are no other limitations except 4 oval-shaped slots for fastening. By the way, we make the material of this side copper, which means that an RFID tag module can be easily welded on the plate.

As shown in Fig. (1), a simple horizontally-etched slotdipole antenna may be taken into consideration. The geometry and parameters of the proposed horizontal slot-dipole antenna for optimization are shown in Fig. (2). In the simulation, the e-plate is located in $y-z$ plane, and the coordinate origin is set at the center of the e-plate. This horizontal slotdipole antenna is etched in the y-direction. The two feed points across the slot are positioned at the center of the slot (marked on the L1 slot in Fig. 2). The geometric parameters of the slot-dipole antenna W1, L1, H1 are defined as in Fig. (2).

As it is shown in Fig. (3a), the active RFID tag module is attached to the back side of the e-plate. The feed structure of the slot-dipole antenna is shown in Fig. (3b) in details. The ground of the tag module is connected to the copper layer at point on the up side of the horizontal slot. The port for antenna on the circuit board of the module is designed to have 


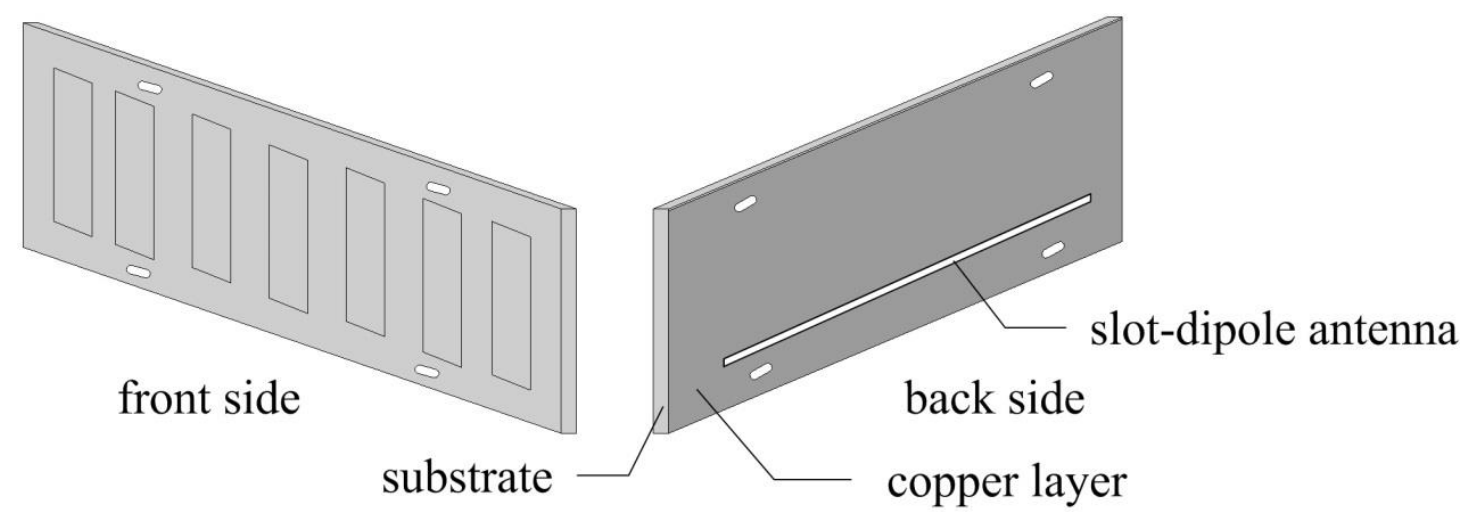

Fig. (1). License plate with a slot-dipole antenna.

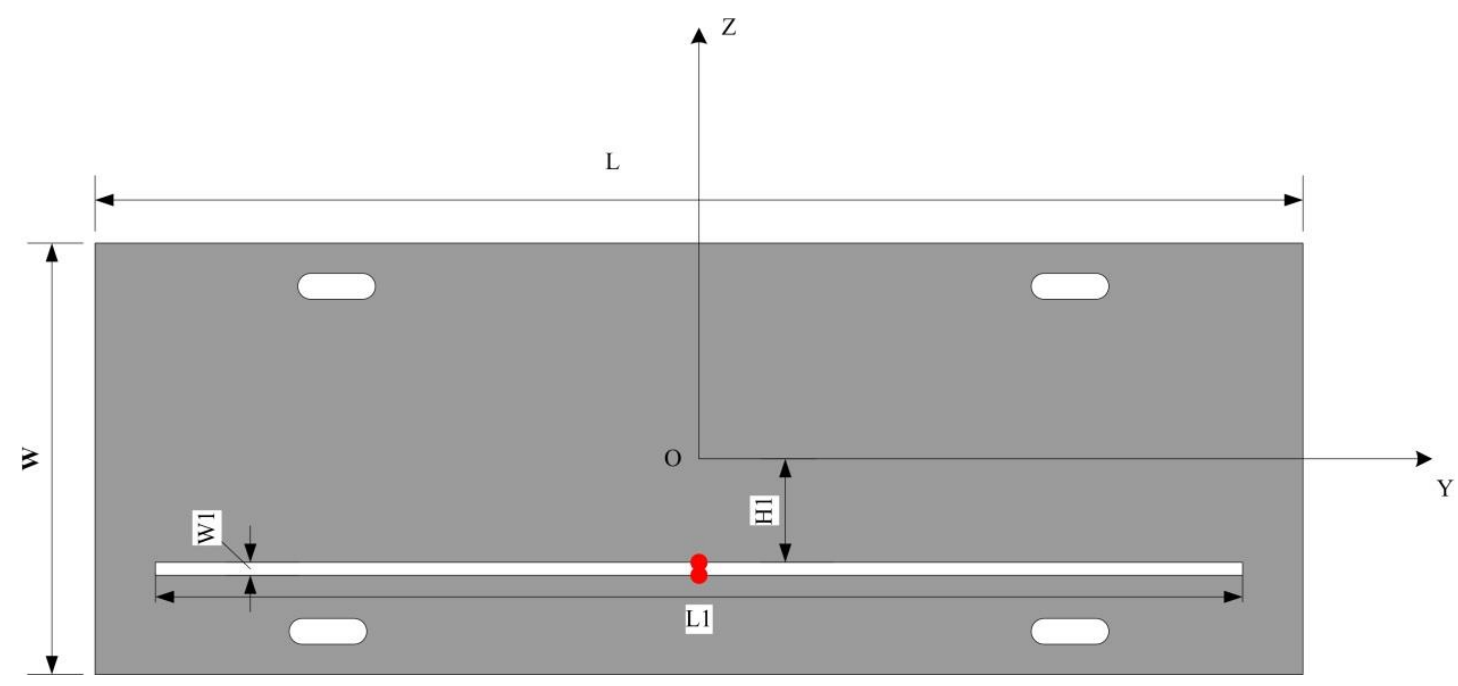

Fig. (2). Geometry and parameters of slot-dipole antenna on e-plate.

a co-planar waveguide (CPW) structure. The strip is extended on the substrate to cross the slot and connected to the copper layer at the feed point on the other side of the horizontal slot, as illustrated in Fig. (3b), where $\mathrm{x} 1$ defines the length of the strip.

There is no doubt that the vehicle metallic structure for the license plate to be mounted will have influence on the performance of the antenna, so a $1320 \times 420 \mathrm{~mm}$ grounded metallic plate behind the e-plate is included in our numerical simulation. Besides, the thickness of the tag circuit with batteries should be taken into account, so the distance between the e-plate and the ground plate is designated up to $8 \mathrm{~mm}$.

\section{SIMULATION RESULTS AND PERFORMANCE}

For demonstration, the proposed e-plate is designed to operate at $2.45 \mathrm{GHz}$. Based on the RFID requirement, there should be an operation bandwidth ranging from $2.4 \mathrm{GHz}$ to $2.483 \mathrm{GHz}$ [7].

The geometric parameters defined in Fig. (2) and Fig. (3b) have been designed and optimized by means of numerical simulations. The resulting optimized values are:

$\mathrm{W} 1=2 \mathrm{~mm}, \mathrm{~L} 1=405 \mathrm{~mm}, \mathrm{H} 1=40 \mathrm{~mm}, \mathrm{x} 1=3 \mathrm{~mm}$.
The computed return loss of the proposed slot-dipole antenna is shown in Fig. (4). The results of simulation show that the proposed slot antenna provides an operation frequency bandwidth of approximately $98 \mathrm{MHz}$, ranging from $2.395 \mathrm{GHz}$ to $2.493 \mathrm{GHz}$. It is obvious that the proposed design has sufficient bandwidth to meet the requirement of the RFID $2.45 \mathrm{GHz}$ system.

To give a clear description of the performance of the eplate, Fig. (5) shows a 3-dimensional radiation pattern of the proposed slot-dipole antenna at the operating frequency of $2.45 \mathrm{GHz}$. We can obviously observe that the proposed eplate has two up-tilted beams directed to the top-right and top-left above the $x-y$ plane. The two beams (phil $=18^{\circ}$ and phi $2=342^{\circ}$ ) are all up-tilt $28^{\circ}\left(\right.$ theta $\left.=62^{\circ}\right)$, and the normalized 2 -dimensional radiation patterns are plotted in Fig. (6a) and (b), respectively.

What's more, the maximum gain of the top-right and the top-left beams are $12.791 \mathrm{dBi}$ and $12.583 \mathrm{dBi}$, respectively. So the maximum gain of the proposed slot-dipole antenna is $12.791 \mathrm{dBi}$, which indicates that the proposed slot-dipole antenna is a high gain antenna.

The above radiation pattern will meet the requirement of the deployment of RFID interrogator in the management of vehicles such as in the Intelligent Transportation Systems 


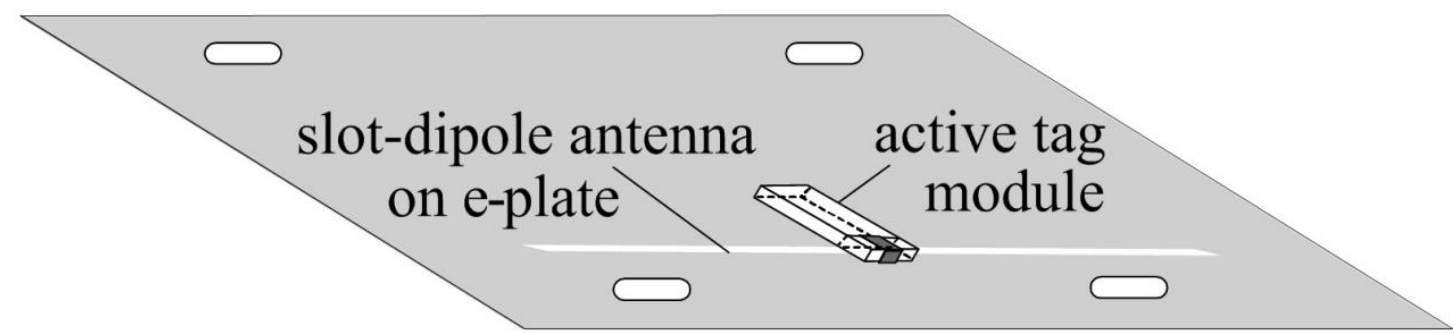

(a) Active RFID tag module on the back

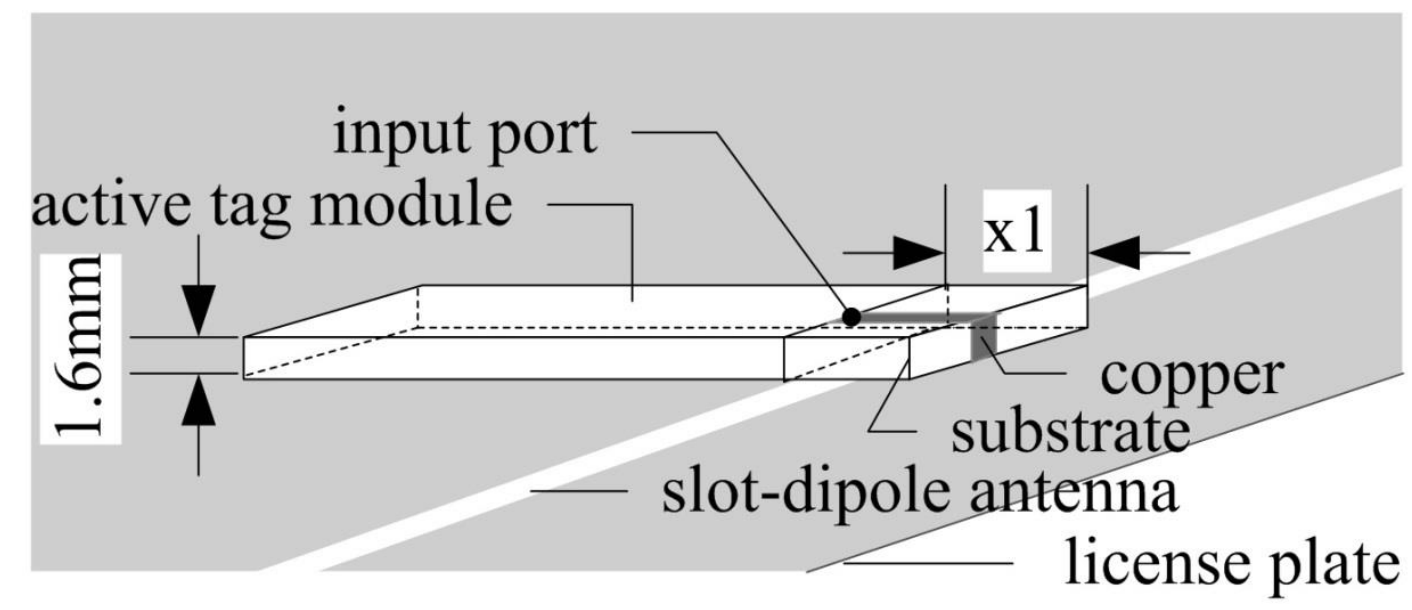

(b) Feed of the slot-dipole antenna

Fig. (3). RFID module and excitation of the slot-dipole antenna.

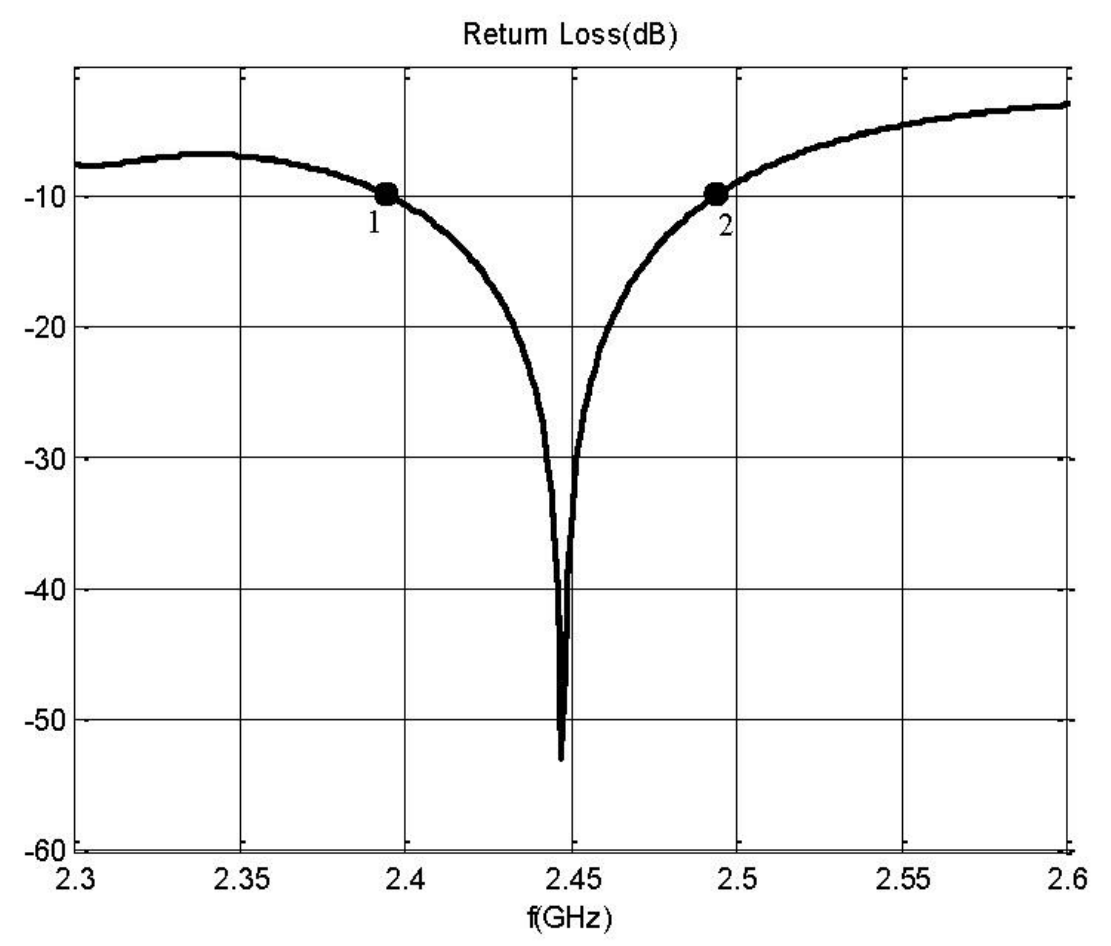

Fig. (4). Simulated return loss for the slot-dipole antenna. 

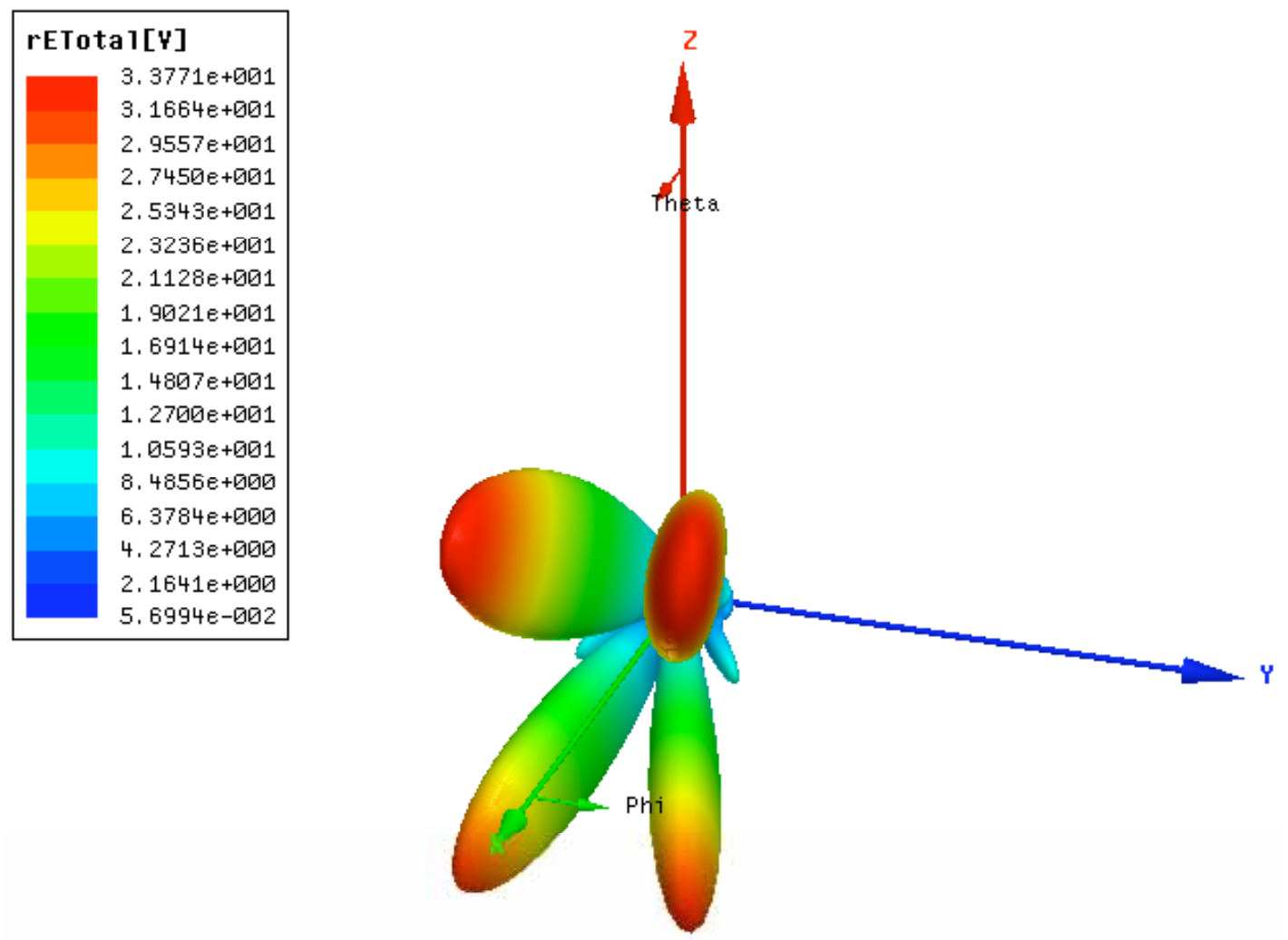

Fig. (5). The three-dimensional radiation pattern of the slot-dipole antenna at $2.45 \mathrm{GHz}$.

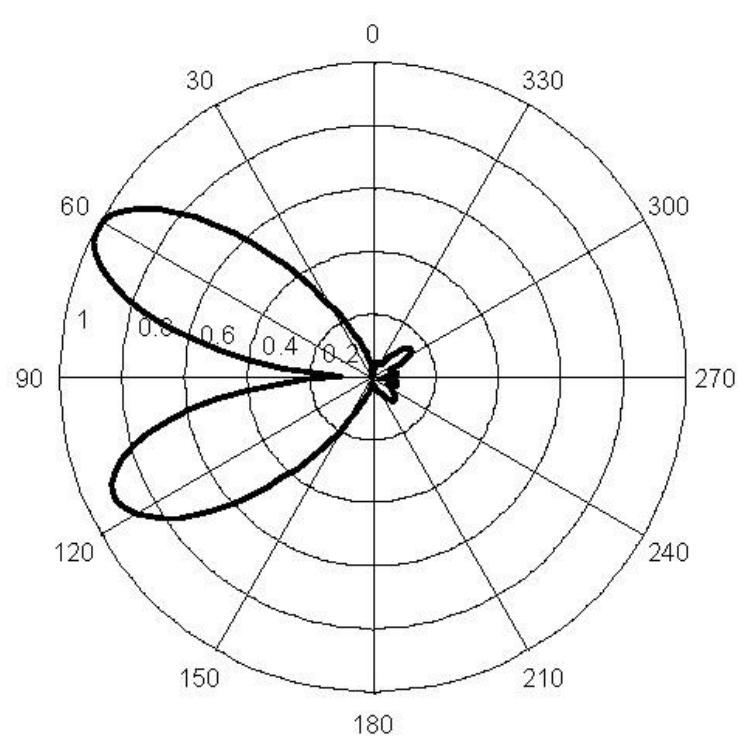

(a) phi1=18deg

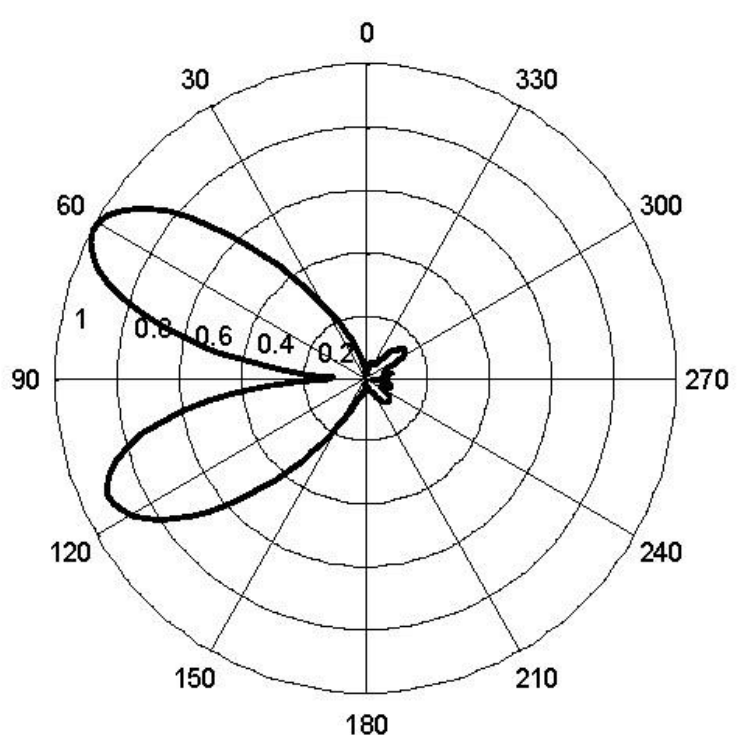

(b) phi2 $=342 \mathrm{deg}$

Fig. (6). The normalized two-dimensional radiation patterns of the slot-dipole antenna at $2.45 \mathrm{GHz}$.

(ITS). For example, if the interrogator can be placed on a lamppost on the roadside, the top-right or the top-left beam will allow the e-plate and interrogator communicate with each other very well.

The proposed e-plate bears an active RFID module which is the same as that we used in the e-plate reported previously in [6]. Therefore, according to the simulated gain and the measured read range in paper [6], the read range of the proposed e-plate in this paper can be estimated readily. is:

The power transmission formula [8] for an RFID system

$$
P_{r}=\left(\frac{\lambda}{4 \pi R}\right)^{2} P_{i} G_{t} G_{r}
$$




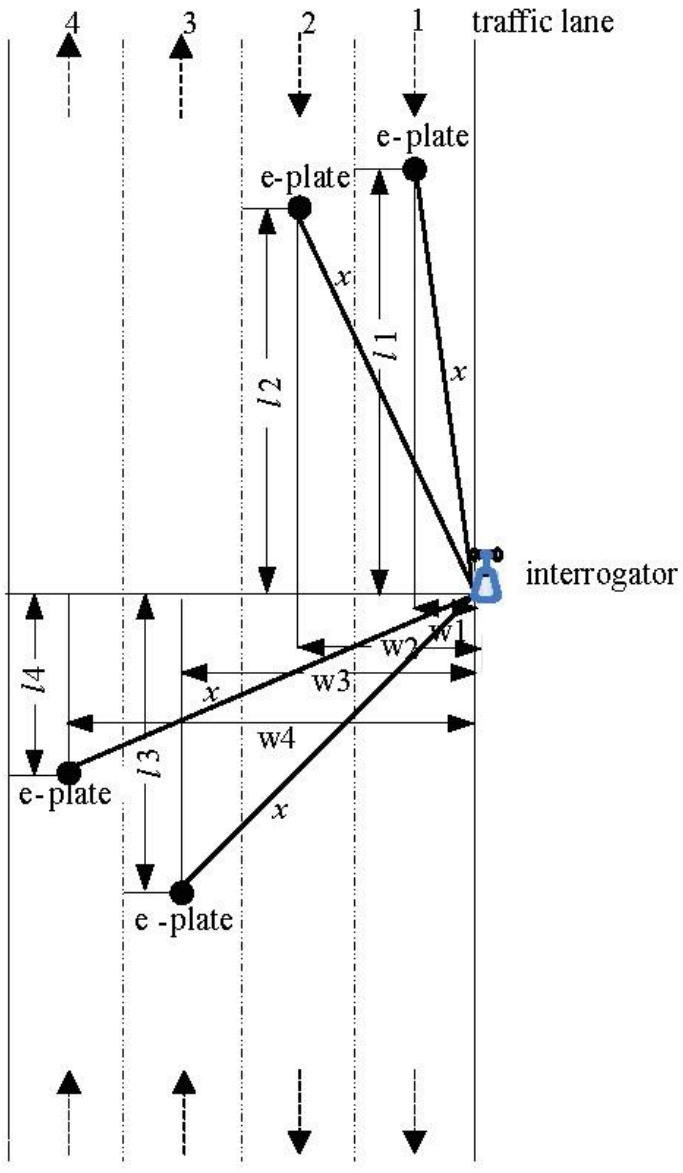

Fig. (7). The read situation of the e-plate when one interrogator is positioned.

where $P_{r}, P_{i}, G_{r}, G_{t}, R$, and $\lambda$ are received power of the receiving antenna, input power of the transmitting antenna, gain of the receiving antenna, gain of the transmitting antenna, distance between the receiving antenna and the transmitting antenna, and wavelength, respectively.

For the e-plate proposed by paper [6], the received power of the reader antenna is:

$P_{r 1}=\left(\frac{\lambda}{4 \pi R_{\text {max }, 1}}\right)^{2} P_{i} G_{t 1} G_{r}$

where $R_{\max , 1}$ is the measured maximum read range, and its value is $92 \mathrm{~m} . G_{t 1}$ is the simulated maximum gain, and its value is $10.683 \mathrm{dBi}$.

For the e-plate proposed by this paper, the received power of the reader antenna is:

$P_{r 2}=\left(\frac{\lambda}{4 \pi R_{\max , 2}}\right)^{2} P_{i} G_{t 2} G_{r}$

where $R_{\max , 2}$ is the estimated maximum read range. $G_{t 2}$ is the simulated maximum gain.

Since both e-plates have the same active RFID tag module, in case they are interrogated by the same RFID interrogator, from Eq. (2) and (3) we can get:

$$
\begin{aligned}
& \left(\frac{\lambda}{4 \pi R_{\text {max }, 1}}\right)^{2} P_{i} G_{t 1} G_{r}=\left(\frac{\lambda}{4 \pi R_{\text {max }, 2}}\right)^{2} P_{i} G_{t 2} G_{r} \\
& \text { i.e., } \\
& \frac{G_{t 2}}{G_{t 1}}=\left(\frac{R_{\text {max }, 2}}{R_{\text {max }, 1}}\right)^{2}
\end{aligned}
$$

The Eq. (5) can be rewritten as:

$$
G_{t 2}(d B)-G_{t 1}(d B)=10 \lg \left(\frac{R_{\text {max }, 2}}{R_{\text {max }, 1}}\right)^{2}
$$

Since the maximum gain of the slot-dipole antenna are $12.791 \mathrm{dBi}$ and $12.583 \mathrm{dBi}$ for the top-right and the top-left beams, respectively, the maximum read range can be calculated as $R_{\max , 2}=114.5 \mathrm{~m}$ when the top-left beam plays the leading role and $R_{\max , 2}=117.3 \mathrm{~m}$ when the top-right beam plays the leading role. In the evaluation, the active RFID tag module is ML-T80, and the interrogator is ML-M800, which are all the same as we used in our previous design in [6]. Judging from the maximum read range, the slot-dipole eplate proposed in this paper meets the read range requirement of e-plate identification system.

\section{DEPLOYMENT AND EFFECT}

For the RFID interrogator deployment of two-way urban road with 4 traffic lanes, first of all, we consider the situation that an interrogator is deployed on the lamppost of height $h$ at the right side of the road, as shown in Fig. (7), which is a top view so as to read the situation of the whole lane easily. It's supposed that the width of each traffic lane is $w$, and its value is $3.75 \mathrm{~m}$, and vehicles are driving in the central part of each traffic lane. For vehicle bearing an e-plate in the forward traffic lanes 1 and 2, the top-right beam plays the leading role. For vehicle bearing the e-plate in the reverse traffic lanes 3 and 4, the top-left beam plays the leading role, and the two beams are all up-tilt $28^{\circ}$.

As shown in Fig. (7), $x$ is defined as the distance of the lamppost to each e-plate, w1 $\sim$ w4 are defined as the widths of the lamp post to the centre of each traffic lane, and $l 1 \sim l 4$ are defined as the optimum read distances of each e-plate. So we obtain:

$$
\begin{aligned}
& x=\frac{h}{\operatorname{tg} 28^{\circ}} \approx 1.887 h(\mathrm{~m}), \\
& l 1=\sqrt{x^{2}-\mathrm{w} 1^{2}}=\sqrt{(1.887 h)^{2}-\left(\frac{w}{2}\right)^{2}}(\mathrm{~m}), \\
& l 2=\sqrt{x^{2}-\mathrm{w} 2^{2}}=\sqrt{(1.887 h)^{2}-\left(\frac{3 w}{2}\right)^{2}}(\mathrm{~m}), \\
& l 3=\sqrt{x^{2}-\mathrm{w} 3^{2}}=\sqrt{(1.887 h)^{2}-\left(\frac{5 w}{2}\right)^{2}}(\mathrm{~m}), \\
& l 4=\sqrt{x^{2}-\mathrm{w} 4^{2}}=\sqrt{(1.887 h)^{2}-\left(\frac{7 w}{2}\right)^{2}}(\mathrm{~m}) .
\end{aligned}
$$




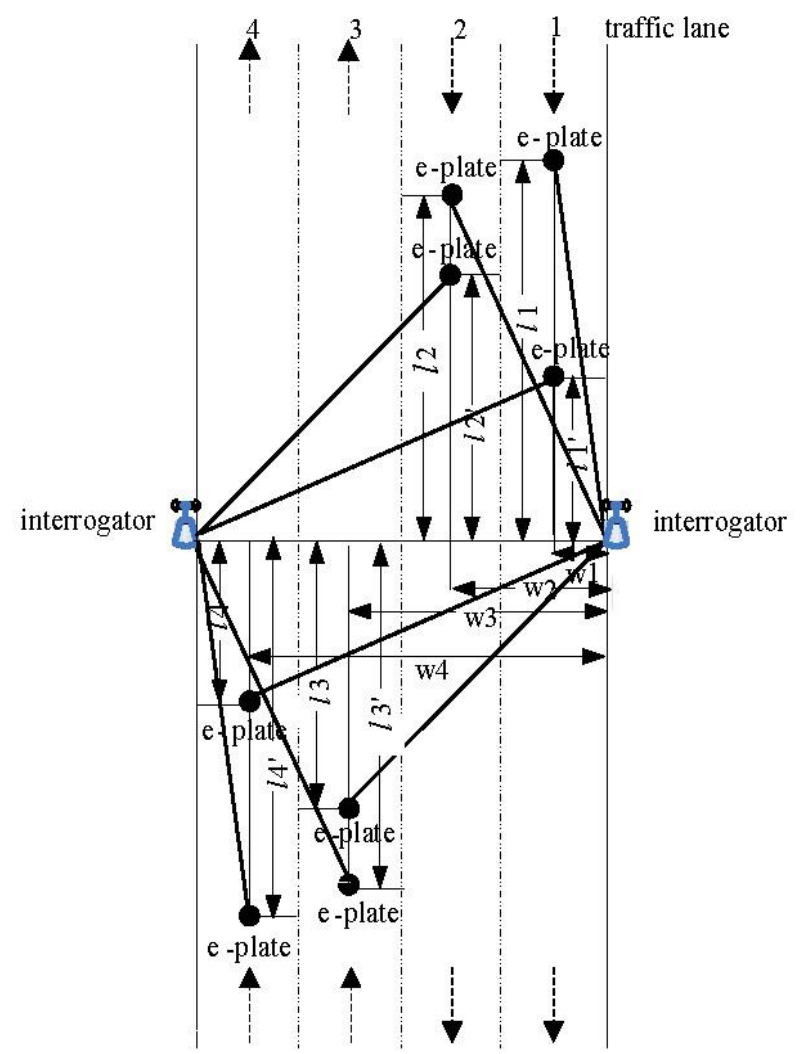

Fig. (8). The read situation of the e-plate when two interrogators are positioned.

Whichever beam plays the leading role, $l 1 \sim l 4$ should not be greater than the maximum estimated read range $R_{\max , 2}$. So we have $l 1 \leq 117.3 \mathrm{~m}$ and $l 3 \leq 114.5 \mathrm{~m}$. Apart from that, the eplate sends signals to the interrogator three times in $1 \mathrm{~s}$. If the driving time in the read range of the e-plate is less than $1 \mathrm{~s}$, the interrogator will probably leak reading. So we have $l 4 / V_{\max } \geq 1 \mathrm{~s}$, where $V_{\max }$ is the maximum speed of vehicle allowed by the road.

From the above we discussed, the height range of the interrogator deployment can be calculated as $\sqrt{\frac{V_{\max }^{2}+\left(\frac{7 w}{2}\right)^{2}}{3.56}} \mathrm{~m} \leq h \leq 61 \mathrm{~m}$. This result shows that the height of interrogator is closely related with $V_{\max }$ and $w$, and when the value of $w$ is fixed, the minimum height of the interrogator increases with the increase of $V_{\max }$. Since we have known that the value of $w$ is $3.75 \mathrm{~m}$, and if $V_{\max }$ is $40 \mathrm{~km} / \mathrm{h}$, we can interrogate the e-plate with the range of $h 9.1 \mathrm{~m} \leq h \leq$ $61 \mathrm{~m}$. If $V_{\max }$ is $60 \mathrm{~km} / \mathrm{h}$, the e-plate can be interrogated with the range of $h 11.2 \mathrm{~m} \leq h \leq 61 \mathrm{~m}$. If $V_{\max }$ is $80 \mathrm{~km} / \mathrm{h}$, the eplate can be interrogated with the aim range: $13.7 \mathrm{~m} \leq h \leq 61$ $\mathrm{m}$. If $V_{\max }$ is $100 \mathrm{~km} / \mathrm{h}$, the e-plate can be interrogated with the aim range: $16.3 \mathrm{~m} \leq h \leq 61 \mathrm{~m}$. If $V_{\max }$ is $120 \mathrm{~km} / \mathrm{h}$, the eplate can be interrogated with the aim range: $19 \mathrm{~m} \leq h \leq 61$ $\mathrm{m}$, and so on.

Second, we consider the situation that two RFID interrogators are deployed on lampposts on the two road sides, as shown in Fig. (8), which is also a top view, where $l 1^{\prime}=l 4$, $l 2^{\prime}=l 3, l 3^{\prime}=l 2, l 4^{\prime}=l 1$. Obviously, each e-plate has two optimum reading positions. If we want to make the minimum height of the interrogator lower than the above we discussed, each interrogator can be responsible for the two traffic lanes of the same direction respectively. So we have $l 1 \leq 114.5 \mathrm{~m}$ and $l 2 / V_{\max } \geq 1 \mathrm{~s}$. And the height range of the interrogator deployment can be calculated as $\sqrt{\frac{V_{\max }{ }^{2}+\left(\frac{3 w}{2}\right)^{2}}{3.56}} \mathrm{~m} \leq h \leq$ $60.6 \mathrm{~m}$. Since the value of $w$ is $3.75 \mathrm{~m}$, and if $V_{\max }$ is $40 \mathrm{~km} / \mathrm{h}$, we can interrogate the e-plate with the range of $h$ $6.6 \mathrm{~m} \leq h \leq 60.6 \mathrm{~m}$. If $V_{\max }$ is $60 \mathrm{~km} / \mathrm{h}$, the e-plate can be interrogated with the range of $h 9.4 \mathrm{~m} \leq h \leq 60.6 \mathrm{~m}$. If $V_{\max }$ is $80 \mathrm{~km} / \mathrm{h}$, the e-plate can be interrogated with the aim range: $12.2 \mathrm{~m} \leq h \leq 60.6 \mathrm{~m}$. If $V_{\max }$ is $100 \mathrm{~km} / \mathrm{h}$, the e-plate can be interrogated with the aim range: $15 \mathrm{~m} \leq h \leq 60.6 \mathrm{~m}$. If $V_{\max }$ is $120 \mathrm{~km} / \mathrm{h}$, the e-plate can be interrogated with the aim range: $18 \mathrm{~m} \leq h \leq 60.6 \mathrm{~m}$, and so on. As we can see above, for the same $V_{\max }$, the minimum height of interrogator deployment in this situation is smaller than that in the situation 1 .

From the two situations we discussed, the position of the RFID interrogator deployment is reasonable. The height and number of the interrogators can be adjusted according to the needs of actual situation.

Also, the RFID interrogator deployment of two-way urban road with 6 traffic lanes or 8 traffic lanes can be analyzed similarly. Obviously, the more the number of the traffic lanes are, the higher the minimum height of the interrogator deployment is. And if there are no suitable lampposts on the roadside, high poles can replace them.

\section{CONCLUSION}

An active e-plate with a simple horizontally-etched slotdipole antenna is designed, and demonstrated by etching slot on a single-sided copper clad plate of dimensions according to Chinese standard license. Performance analysis of the proposed slot-dipole e-plate indicates that the proposed e-plate can meet the requirements for on-road vehicle identification when the RFID interrogators are deployed on the roadside lampposts. The height and number of the interrogators can be adjusted according to the maximum speed of vehicle allowed by the road, the width of each traffic lane, and the number of the traffic lanes.

\section{CONFLICT OF INTEREST}

The authors confirm that this article content has no conflicts of interest.

\section{ACKNOWLEDGEMENTS}

Declared none.

\section{REFERENCES}

[1] e-Plate Ltd., "e-Plate Presentation", [Online]. Available: www.eplate.com/technical.html [Accessed 4th Nov. 2013].

[2] H. Bantli, E. J. Ring, and W. M. Thomas, "Electronic license plate architecture,” U.S. Patent No. 5,608,391, March 4, 1997. 
[3] W. Mish, "Vehicles, license plate frame assemblies, methods of forming license plate frames, and methods of attaching RFIDs, transponders and modulators to vehicle," U.S. Patent No. 6,025,784, February 15, 2000.

[4] L. Mo, H. Zhang, and H. Zhou, "Broadband UHF RFID tag antenna with a pair of U slots mountable on metallic objects," Electronics Letter, vol. 44, no. 20, pp. 1173-1174, 2008.

[5] Y. Um, U. Kim, and J. Choi. "Design of a compact CPW-fed UHF RFID tag antenna for metallic objects," Microwave and Optical Technology Letters, vol. 50, no. 5, pp. 1439-1443, 2008.
[6] W. J. Zhao, G. Wang, and X. Lai, "Active E-plate with slot antenna," IEEE Int. Conf. on Wireless Communications, Networking and Mobile Computing, pp.1-3, 2008.

[7] K. Finkenzeller, RFID Handbook: Fundamental and Applications in Contactless Smart Cards and Identification, $3^{\text {rd }}$ ed. John Wiley \& Sons, 2010.

[8] J. D. Kraus and R. J. Marhefka, Antennas: For All Applications, $3^{\text {rd }}$ ed. McGraw-Hill, 2003.

Received: January 09, 2014

(C) Zhao et al.; Licensee Bentham Open.

This is an open access article licensed under the terms of the Creative Commons Attribution Non-Commercial License (http://creativecommons.org/licenses/by-nc/3.0/) which permits unrestricted, non-commercial use, distribution and reproduction in any medium, provided the work is properly cited. 